\title{
The New Bioethics: Reintegration of Environmental and Biomedical Sciences
}

This special section is an expansive discussion of a wide swath of bioethical topics in the two most important public health professions: engineering and medicine. In fact, a number of the articles address what have become known as the "environmental health professions." Engineers have designed systems to clean the air and water, to improve the safety of food, and to protect natural resources. Physicians and medical practitioners have treated and prevented diseases and are increasingly promoting the wellness of individuals and populations. A variety of environmental health scientists are helping to bridge these two disparate fields. Obviously, physicians and medical practitioners are the healers of our time. Unfortunately, few endeavors in the life sciences are without some costs and tradeoffs. This is the province of ethical decision making.

The term "bioethics" has lost some of its meaning since it was coined by Van Rensselaer Potter II (1911-2001) in the 1970s. It is now generally assumed to be a synonym for biomedical ethics, but the term originally conveyed a sense of integration and systematic thinking in all decisions related to living things. Thus, Ethics in Biology, Engineering, and Medicine is the ideal venue for retracing and reconstructing bioethics back to its comprehensive roots, which encompassed moral decision making regarding both medicine and the environment.

Potter considered bioethics as a bridge between the sciences and the humanities to serve the best interests of human health and to protect the environment:

From the outset it has been clear that bioethics must be built on an interdisciplinary or multidisciplinary base. I have proposed two major areas with interests that appear to be separate but which need each other: medical bioethics and ecological bioethics. Medical bioethics and ecological bioethics are non-overlapping in the sense that medical bioethics is chiefly concerned with short-term views: the options open to individuals and their physicians in their attempts to prolong life.... Ecological bioethics clearly has a long-term view that is concerned with what we must do to preserve the ecosystem in a form that is compatible with the continued existence of the human species. ${ }^{1}$

This issue of EBEM includes a diverse group of authors who consider ethical decision making from numerous perspectives. Both the content and the conduct of science are addressed. What are some of the unique challenges of environmental research that involves human subjects? Are current investigations and conclusions adhering to wellestablished norms or are they drifting toward advocacy? Conversely, are we properly including all or even the correct members of the community so that our research is relevant and useful? Are the methods being employed ensuring good science or are there weaknesses due to conflicts of interest? Is privacy sacrificed inappropriately? How well are we teaching ethics within scientific subject matter, and what approaches would work 
better? Are we truly respecting persons and providing proper autonomy, the best means of protecting health, and livable environments?

The march of the biological sciences has been justified as an overall benefit to humankind. However, this commitment and involvement calls for deliberate and serious considerations of actual and potential ethical issues. The President's Council on Bioethics $^{2}$ has summarized the dichotomy between the promise and ethical challenges:

... knowledge of how things work often leads to new technological powers to control or alter these workings, powers generally sought in order to treat human disease and relieve suffering. But, once available, powers sought for one purpose are frequently usable for others. The same technological capacity to influence and control bodily processes for medical ends may lead (wittingly or unwittingly) to non-therapeutic uses, including 'enhancements' of normal life processes or even alterations in 'human nature.' Moreover, as a result of anticipated knowledge of genetics and developmental biology, these transforming powers may soon be able to transmit such alterations to future generations.

Human health is inextricably tied to the environment. Therefore, the ethics of the life sciences must also be considered systematically in the search for proper means of intervention and prevention. Certainly, some of the challenges of medical practitioners and researchers are unique to their specialties. Neurosurgeons must make decisions about cognition, for example, when deciding on whether treating a disease is worth changes in personhood. However, many ethical challenges are shared by all life scientists. After all, medicine and engineering are working toward the same general objective: healthy people living in a healthful environment. Both professions apply the sciences to achieve this objective, albeit at different scales and complexity (e.g., physicians deal with one species and environmental scientists address many species). In this sense, this issue is all about ecology in a rather broad context. The authors are looking at how the life sciences can be used within the boundaries established by the scientific method to improve the public's health and welfare and how this can be done both morally and practically.

Some of the connections are rather obvious, such as the need for credible environmental studies of exposures of children to lead and mercury coupled with sound medical diagnosis and treatment of neurological problems, all the while respecting families and communities. This calls for analyzing the data in such a way as to assist the engineer and others in reducing or preventing exposure and changing the materials used in a product. Other connections are more indirect, or even obscure. For example, what will be the role of medical researchers and practitioners if climate change leads to the migration of tropical diseases? How certain must we be about the science before actions are taken to reduce the probability of expanded disease incidence?

Environmental and biomedical ethics are complicated because life is complicated. I tease my fellow engineers who happen to work in more abiotic disciplines (e.g., structural) that they enjoy much higher levels of precision and less uncertainty than those of us in the "bio" disciplines. Living systems are chaotic and messy. 
Another challenge of life science ethics is that precaution does not precisely equal morality. In some cases, assuming the worst case is not tantamount to taking the ethical high ground. Unlike physicians, who are all in the business of biology, engineers may think of themselves as being in the business of physics, with the biomedical and environmental disciplines targeting this physics at living systems. While this is arguably true, all engineering is also "biotic" to some extent. Indeed, the engineer's principal client is the public, so the structure must not only stand, it must serve a particular function. Every discipline must employ human factors engineering - not just answering the question of how something should be used, but also how it might be used. Do not be surprised at some of the novel (and dangerous) ways that something you design will be used, other than what you had thought would be its function. ${ }^{3}$ The point here is that outcomes will seldom follow a nice, linear path to the desired outcome. Sometimes, even a seemingly small, unaccounted for factor could result in an outcome no one expected or wanted. For example, if climate change is assumed to be drastic and calls for strict reductions in greenhouse gas emissions, could this stifle economic development and limit the opportunities for people to be lifted out of poverty? If we take a product off the market because it may damage the environment, but it is the only effective treatment of a debilitating disease, how did we weigh the risks and benefits to prevent greater human suffering?

This issue is devoted to considering such challenges in a systematic way. As is so often the case in ethical inquiry, there are many questions left unanswered. In fact, we may well have introduced more than we have resolved. I hope that reading these eclectic articles helps you to join the dialogue, and to extend it to all of the practitioners and researchers in the life sciences. Protecting human health and environmental quality calls for a proper consideration of attendant moral decisions. That is the goal of this EBEM issue. I would like to think that Potter would have agreed and may well have contributed.

\section{REFERENCES}

1. Potter II VR. What does bioethics mean? The Ag Bioethics Forum.1996;8(1):2-3. Available from: http://www.bioethics.iastate.edu/forum/jun.96pg2.html.

2. The President's Council on Bioethics, Department of Health \& Human Services. Discussion of cloning (Working Paper 1, Session 4: Human cloning 1: human procreation and biotechnology). 17-2001 Jan 18; Washington, DC.

3. Vallero DA. Biomedical ethics for engineers: Ethics and decision making in biomedical and biosystem engineering. Amsterdam: Academic Press; 2007.

Daniel A. Vallero, Ph.D. Civil and Environmental Engineering

Adjunct Professor of Engineering Ethics

Duke University, Pratt School of Engineering

Durham, NC 27708 